\title{
IDENTIFICAÇÃO DE ERROS DE TOPOLOGIA VIA MULTIPLICADORES DE LAGRANGE NORMALIZADOS E TESTES DE HIPÓTESES
}

\author{
Elizete Maria Lourenço* \\ elizete@eletrica.ufpr.br
}

\author{
Antonio Simões Costa ${ }^{\dagger}$ \\ simoes@labspot.ufsc.br
}

*Departamento de Engenharia Elétrica, Universidade Federal do Paraná, Curitiba, PR, Brasil

${ }^{\dagger}$ Departamento de Engenharia Elétrica, Universidade Federal de Santa Catarina 66040-900 - Florianópolis, SC, Brasil

\begin{abstract}
This paper proposes the use of hypothesis testing for topology error identification based on normalized Lagrange multipliers. The main objective is to avoid state re-estimations that would be otherwise required for each possible set of suspect breaker statuses. The hypotheses are defined in terms of the set of circuit breakers whose statuses are under suspicion. The proposed hypothesis testing procedure relies on Bayes' theorem to compute the conditional probabilities for the hypotheses, which are then used to identify the correct network topology.
\end{abstract}

KEYWORDS: Identification of topology errors in power networks, power system state estimation; power system real time modeling.

\section{RESUMO}

Este artigo desenvolve uma metodologia baseada em testes de hipóteses a ser associada à técnica de identificação de erros topológicos via multiplicadores de Lagrange normalizados recentemente proposta na literatura. O principal objetivo é eliminar a necessidade de re-estimação de estados a cada nova configuração a ser testada, requerida pelo método citado. As hipóteses são

Artigo submetido em $19 / 12 / 00$

1a. Revisão em 13/11/01; 2a. Revisão em 25/04/02; 3a. Revisão em $15 / 07 / 02$

Aceito sob recomendação do Ed. Assoc. Prof. José L. R. Pereira definidas pelos status dos disjuntores suspeitos de terem sido erroneamente modelados. Os testes de hipóteses propostos aplicam o teorema de Bayes de modo a obter como resultado probabilidades condicionais para as hipóteses. A partir destas, é possível identificar a correta configuração da rede.

PALAVRAS-CHAVE: Identificação de erros de topologia em sistemas de potência, estimação de estados em sistemas de potência, modelagem em tempo real de sistemas de potência

\section{INTRODUÇÃO}

A modelagem em tempo real é uma ferramenta fundamental na moderna operação de sistemas elétricos de potência. Vários aplicativos destinados à análise da segurança da operação dependem da disponibilidade de modelos confiáveis obtidos a partir do processamento de informações adquiridas em tempo real. Um requisito para a obtenção de tais modelos é o conhecimento da topologia da rede que corresponde às condições efetivamente existentes, definidas pelas informações sobre o status de chaves e disjuntores. Erros nestes dados comprometem os resultados fornecidos pelos aplicativos executados subseqüentemente, tais como a estimação de estados, a análise de contingências e o fluxo de potência em tempo real.

A busca de métodos eficientes de identificação de er- 
ros de topologia na estimação de estado tem inspirado diversas pesquisas. Algumas destas propõem o uso de sistemas especialistas (Singh e Glavitsch, 1991), (Singh e Oesch, 1994), (Souza et alli, 1998) para detectar e identificar erros de topologia.

Desenvolvimentos recentes relacionados à representação dos ramos de impedância nula (Monticelli e Garcia, 1991), (Monticelli, 1993) e à possibilidade do uso de multiplicadores de Lagrange na detecção de erros grosseiros e erros topológicos (Gjelsvik, 1993), renovaram o interesse de pesquisa nesta área. Neste sentido, novos algoritmos de estimação de estados (Alsaç et alii , 1998) e de identificação de erros topológicos (Clements e Simões Costa, 1997), (Abur et alli, 1995), foram propostos recentemente.

Este trabalho propõe a associação de testes de hipóteses baseados no Teorema de Bayes à identificação de erros topológicos via multiplicadores de Lagrange normalizados, com o objetivo de eliminar a necessidade de re-estimação dos estados a cada nova configuração a ser testada, requerida em (Clements e Simões Costa, 1997).

No método proposto neste artigo, os multiplicadores de Lagrange normalizados são utilizados para determinar os disjuntores suspeitos de conterem erro de modelagem. Os testes de hipóteses fornecem como resultado a probabilidade condicional para as combinações de status destes disjuntores, possibilitando ao final a identificação da correta configuração da rede.

\section{ESTIMAÇÃO DE ESTADOS COM RES- TRIÇÕES E COM INFORMAÇÕES A PRIORI}

\subsection{Modelagem de Ramos de Impedância Nula: Restrições Operacionais}

O método proposto utiliza a representação detalhada de regiões da rede previamente identificadas como suspeitas. Estas regiões são modeladas no nível de seção de barra. Para isto, a representação de disjuntores proposta em (Monticelli e Garcia, 1991) e (Monticelli, 1993) é utilizada, segundo a qual os fluxos ativos e reativos através dos disjuntores são incluídos como variáveis de estado. Conseqüentemente, para representar um disjuntor entre os nós $i$ e $j$, os fluxos de potência ativa $t_{i j}$ e reativa $u_{i j}$ são incluídos no vetor de estados.

Para representar um dado disjuntor fechado, a diferença angular $\delta_{i}-\delta_{j}$ e a queda de tensão $V_{i}-V_{j}$ são consideradas nulas. Se o disjuntor estiver aberto, os fluxos ativo $t_{i j}$ e reativo $u_{i j}$ serão iguais a zero. Estas infor- mações são inseridas no modelo matemático sob forma de restrições ao problema de estimação de estados. Tais restrições são chamadas de restrições operacionais, uma vez que um disjuntor pode ter seu status modificado de acordo com necessidades ditadas pelas condições de operação (Clements e Simões Costa, 1997), e serão referenciadas genericamente na formulação da estimação de estados restrita por:

$$
h_{o}(x)=0
$$

onde $h_{o}(x)$ é um vetor $N_{o} \times 1$ de funções não-lineares que representam as restrições operacionais e $x$ é o vetor $n \times 1$ de variáveis de estado, contendo tanto as variáveis nodais convencionais quanto os fluxos de potência ativa e reativa pelos disjuntores.

\subsection{Restrições Estruturais}

Além das restrições operacionais, outras restrições podem surgir a partir da configuração física da rede. Em particular, pode-se citar as barras/nós de passagem, as quais obedecem as restrições de injeção de potência ativa e reativa nulas, ou seja, para a barra/nó de passagem $i$, $p_{i}=0$ e $q_{i}=0$. A escolha de uma barra $i$ como referência angular para o sistema também pode ser modelada como uma restrição, sendo esta do tipo $\delta_{i}=0$. Estas restrições são chamadas restrições estruturais e serão genericamente referenciadas por:

$$
h_{s}(x)=0
$$

onde $h_{s}(x)$ é um vetor $N_{s} \times 1$ de funções não-lineares que representam as restrições estruturais.

\subsection{Modelagem das Telemedidas Analógi- cas}

Adicionalmente, o modelo em tempo real deve incluir as telemedidas analógicas provenientes do sistema de aquisição de dados. Estas são modeladas da seguinte forma:

$$
\begin{gathered}
z_{m}=h_{m}(x)+\varepsilon_{m} \\
E\left\{\varepsilon_{m} \varepsilon_{m}^{T}\right\}=R_{m}
\end{gathered}
$$

onde $h_{m}(x)$ é o vetor $m \times 1$ de funções das medidas, $\varepsilon_{m}$ é o vetor de mesma dimensão contendo os erros de medição e $R_{m}$ é a matriz $m \times m$ de covariância dos erros de medição. 


\subsection{Formulação da Estimação de Estados com Restrições e com Informações A Priori}

O problema de estimação de estados consiste na minimização da soma ponderada dos quadrados dos resíduos de estimação. Considerando a incorporação das restrições operacionais e estruturais, o problema passa a ser de otimização restrita, e será descrito por:

$$
\begin{array}{ll}
\text { Minimizar } & \frac{1}{2} r_{m}^{T} R_{m}^{-1} r_{m} \\
\text { Sujeito a: } & -r_{m}+z_{m}-H_{m} \hat{x}=0 \\
& h_{s}(\hat{x})=0 \\
& h_{o}(\hat{x})=0
\end{array}
$$

onde:

$r_{m}$ : vetor $m \times 1$ de resíduos das medidas e $\hat{x}$ : vetor $n \times 1$ de estimativas para os estados.

No método proposto, informações a priori eventualmente disponíveis sobre as variáveis de estado podem ser incluídas no problema de estimação de estados. Estas informações são modeladas diretamente na função objetivo do problema de otimização (5), que é acrescida do termo:

$$
\frac{1}{2}(\hat{x}-\bar{x})^{T} P^{-1}(\hat{x}-\bar{x})
$$

onde $\bar{x}$ é um vetor $n \times 1$ que contém as informações $a$ priori para os estados, cuja incerteza é modelada através de uma matriz $n \times n$ de covariância $P$. Caso não se tenha melhores informações sobre os valores a priori para os ângulos das tensões na barras $\theta$, pode-se assumir que estes são iguais a zero radianos, isto é $\bar{x}_{\theta_{i}}=0 \mathrm{rad}$. Para definir os valores correspondentes na matrix de covariância $P$, considera-se que os valores a priori para os ângulos, $\bar{x}_{\theta_{i}}$, são não-correlacionados e uniformemente distribuídos no intervalo $\left[-\theta_{\lim }, \theta_{\lim }\right]$, onde $\theta_{\text {lim }}$ estabelece os limites para $\theta$ sob as condições de estabilidade em regime permanente (por exemplo, $\theta_{\lim }=\pi / 2 \mathrm{rad}$ ). Um procedimento similar pode ser adotado para os valores a priori das demais variáveis de estado.

Levando em consideração as informações a priori e a representação explícita dos disjuntores, o problema de estimação de estados, torna-se:

$$
\begin{array}{ll}
\text { Minimizar } & \frac{1}{2} r_{m}^{T} R_{m}^{-1} r_{m}+\frac{1}{2}(\hat{x}-\bar{x})^{T} P^{-1}(\hat{x}-\bar{x}) \\
\text { Sujeito a: } & -r_{m}+z_{m}-h_{m}(\hat{x})=0 \\
& h_{s}(\hat{x})=0 \\
& h_{o}(\hat{x})=0
\end{array}
$$

As condições necessárias para a solução ótima do sistema representado pela equação (7) podem ser expressas em termos da seguinte função Lagrangeana:

$$
\begin{aligned}
\mathcal{L}=\frac{1}{2} r_{m}^{T} R_{m}^{-1} r_{m}+ & \frac{1}{2}(\hat{x}-\bar{x})^{T} P^{-1}(\hat{x}-\bar{x}) \\
+\lambda_{m}( & \left.-r_{m}+z_{m}-h_{m}(\hat{x})\right) \\
& +\lambda_{s}\left(-h_{s}(\hat{x})\right)+\lambda_{o}\left(-h_{o}(\hat{x})\right)
\end{aligned}
$$

Aplicando-se o método de Newton para obter a solução do sistema de equações resultante das condições de otimalidade de Karush-Kuhn-Tucker (KKT) correspondentes à função Lagrangeana (8), obtém-se:

$$
\begin{aligned}
\left(\begin{array}{cccc}
-P^{-1} & H_{m}^{T} & H_{s}^{T} & H_{o}^{T} \\
H_{m} & R_{m} & 0 & 0 \\
H_{s} & 0 & 0 & 0 \\
H_{o} & 0 & 0 & 0
\end{array}\right) & \left(\begin{array}{c}
\Delta \hat{x} \\
\lambda_{m} \\
\lambda_{s} \\
\lambda_{o}
\end{array}\right) \\
& =\left(\begin{array}{c}
-P^{-1}\left(\bar{x}-\hat{x}^{(k)}\right) \\
z_{m}-h_{m}\left(\hat{x}^{(k)}\right) \\
h_{s}\left(\hat{x}^{(k)}\right) \\
h_{o}\left(\hat{x}^{(k)}\right)
\end{array}\right)
\end{aligned}
$$

onde $H_{m}, H_{s}$ e $H_{o}$ são as matrizes Jacobianas das funções $h_{m}(\hat{x}), h_{s}(\hat{x})$ e $h_{o}(\hat{x})$, de dimensões $m \times n, N_{s} \times n$ e $N_{o} \times n$, respectivamente, e $\lambda_{m}, \lambda_{s}$ e $\lambda_{o}$ são os multiplicadores de Lagrange associados às medidas, às restrições estruturais e às restrições operacionais, respectivamente. Note que a função Lagrangeana, representada pela equação (8), é linear em $\lambda$. Portanto, só é necessário se proceder à linearização do problema com relação a variável $\hat{x}$, ou seja, a única variável incremental no vetor de incógnitas da equação (9) é $\Delta \hat{x}$.

Por conveniência, definimos o vetor $n m r \times 1$ de multiplicadores de Lagrange como:

$$
\lambda \triangleq\left[\begin{array}{lll}
\lambda_{m}^{T} & \lambda_{s}^{T} & \lambda_{o}^{T}
\end{array}\right]^{T}
$$

onde:

$$
n m r \triangleq m+N_{s}+N_{o}
$$

Finalmente, o problema de estimação de estados restrita com informações a priori (7) pode ser resolvido usando o algoritmo do tableau esparso de Hachtel (Gjelsvik et alli, 1985) que soluciona o sistema linear descrito em (9).

Além de $\lambda_{m}$, o vetor $\lambda$ contém os multiplicadores de Lagrange associados às restrições estruturais e operacionais. Os valores destes multiplicadores representam a sensibilidade da função objetivo $J(\hat{x})$ com relação a variações nestas restrições (Gjelsvik, 1993). 


\section{TESTES DE HIPÓTESES PARA A IDENTIFICAÇÃO DE ERROS DE TO- POLOGIA}

\subsection{Multiplicadores de Lagrange Normali- zados}

O multiplicador de Lagrange normalizado $\lambda_{i}^{N}$ é definido em (Clements e Simões Costa, 1997) como:

$$
\lambda_{i}^{N} \triangleq \frac{\lambda_{i}}{\sqrt{W_{i i}}}
$$

onde $W$ é a matriz de covariância de $\lambda$. Pode-se demonstrar que, na ausência de erros grosseiros em medidas e supondo que as restrições modelam corretamente a rede, os multiplicadores de Lagrange $\lambda$ são variáveis aleatórias de média zero (Clements e Simões Costa, 1997). Portanto, sob as mesmas condições, o multiplicador de Lagrange normalizado $\lambda_{i}^{N}$ é uma variável aleatória de média zero e variância unitária. Além disso, verificase que os multiplicadores de Lagrange normalizados referentes às medidas, $\lambda_{m}^{N}$, são equivalentes aos resíduos normalizados. Os multiplicadores de Lagrange normalizados fornecem uma ferramenta para a deteç̧ão e identificação de erros em restrições, da mesma forma que os resíduos normalizados são utilizados na detecção e identificação de erros grosseiros em medidas (Clements e Simões Costa, 1997). Desta última referência, pode-se ainda verificar que, se os erros de medição apresentam distribuição normal, o mesmo ocorrerá com os multiplicadores de Lagrange $\lambda$, de modo que, na ausência de erros grosseiros e de topologia, $\lambda_{i}^{N}$ apresenta distribuição normal unitária.

A matriz $W$ pode ser obtida a partir da esparsa inversa da matriz de coeficientes da equação (9):

$$
\left(\begin{array}{ll}
-\sum & C^{T} \\
C & W
\end{array}\right) \triangleq\left(\begin{array}{ll}
0 & H^{T} \\
H & R
\end{array}\right)^{-1}
$$

onde:

$$
H \triangleq\left(\begin{array}{c}
H_{m} \\
H_{s} \\
H_{o}
\end{array}\right) ; R \triangleq\left(\begin{array}{ccc}
R_{m} & 0 & 0 \\
0 & 0 & 0 \\
0 & 0 & 0
\end{array}\right)
$$

e $\Sigma, C$ e $W$ são partições da inversa do lado direito da equação (13), com dimensões correspondentes às de $P^{-1}, H$ e $R$, respectivamente.

\subsection{Seleção de Disjuntores Suspeitos}

É suposto que os resultados obtidos a partir de uma estimação de estados previamente realizada são utilizados para delimitar as regiões da rede prováveis de conterem erros topológicos, de tal forma que apenas algumas substações necessitem ser detalhadas no nível de seção de barras. Ainda assim, dependendo da dimensão do sistema e do número de substações a serem detalhadas, aplicar testes de hipóteses para a identificação de erros topológicos a todas as possíveis combinações dos status de disjuntores da rede pode resultar em um esforço computacional elevado.

No método proposto, os multiplicadores de Lagrange normalizados são utilizados para detectar erros de topologia e selecionar os disjuntores suspeitos de conterem erro de modelagem. Seja $\left|\lambda_{o_{-} \max }^{N}\right|$ o máximo multiplicador de Lagrange normalizado associado às restrições operacionais. Utilizando o fato de que, na ausência de erros de topologia e de erros grosseiros, $\lambda_{o}^{N}$ tem distribuição normal unitária, o seguinte critério pode ser usado para se concluir sobre a existência de erros de topologia:

$$
\left|\lambda_{o_{-} \max }^{N}\right|>\lambda_{t}
$$

onde $\lambda_{t}$ é um limiar pré-especificado. Como a probabilidade do valor de uma variável aleatória com distribuição normal unitária estar entre $-3 \sigma$ e $+3 \sigma$ é de $99 \%$ (Papoulis, 1991), um valor típico para $\lambda_{t}$ é 3,0.

No caso em que a existência de erro de topologia se verifique, os disjuntores associados às restrições operacionais cujos multiplicadores de Lagrange normalizados $\left(\lambda_{o, i}^{N}\right)$ tem magnitude superior ao limiar $\lambda_{t}$ são selecionados como suspeitos. Os testes de hipóteses são então aplicados às combinações formadas pelos status do conjunto de disjuntores selecionados como suspeitos.

\subsection{Definição das Hipóteses}

Se $n_{d s}$ disjuntores forem selecionados como suspeitos no processo descrito na seção anterior, então existirão $2^{n_{d s}}$ possíveis combinações de status para este conjunto de disjuntores. A hipótese básica $\mathcal{H}_{0}$ é definida como sendo a combinação de status dos disjuntores suspeitos originalmente considerada. Cada uma das demais combinações de status possíveis para o conjunto de disjuntores suspeitos estabelece uma hipótese alternativa $\mathcal{H}_{i}$.

Os estados do sistema são estimados considerando a atual configuração da rede, correspondente à hipótese básica. Os resultados obtidos com esta estimação são também utilizados para o processamento das hipóteses alternativas. Evita-se assim, o esforço computacional de se realizar uma nova estimação de estados para cada hipótese alternativa. Os detalhes dos procedimentos computacionais correspondentes são descritos nas subseções que seguem. 


\subsubsection{Hipótese Básica $-\mathcal{H}_{0}$}

Para que seja possível obter as informações necessárias para cada hipótese alternativa a partir dos resultados da estimação de estados para a hipótese básica, tanto as restrições operacionais para posição aberta quanto para a posição fechada de cada disjuntor devem ser representadas no problema de estimação de estados (7), independentemente do atual status do disjuntor. Para representar a atual configuração da rede neste caso, é necessário que as restrições operacionais que não representam a atual posição dos disjuntores, daqui por diante referenciadas como restrições complementares, sejam desativadas. As restrições complementares são desativadas através de sua transformação em "medidas" de variância elevada, o que afetará os valores diagonais correspondentes da matriz $R$.

\subsubsection{Hipóteses Alternativas $-\mathcal{H}_{i}$}

Para uma hipótese alternativa $\mathcal{H}_{i}$, o status de pelo menos um disjuntor suspeito tem posição diferente daquela apresentada pela hipótese básica. Para representar a configuração estabelecida por $\mathcal{H}_{i}$ a partir de $\mathcal{H}_{0}$, é necessário desativar as restrições operacionais de posição aberta e ativar as restrições operacionais de posição fechada para os disjuntores suspeitos cujo status é fechado em $\mathcal{H}_{i}$ e aberto em $\mathcal{H}_{0}$, e vice-versa para os disjuntores suspeitos cujo status é aberto em $\mathcal{H}_{i}$ e fechado em $\mathcal{H}_{0}$. A representação de cada hipótese alternativa é baseada em modificações na matriz de covariância $R$ que, conforme descrito na subseção 3.3.1, podem ser representadas por:

$$
R^{i}=R+\Delta R^{i}
$$

onde:

$$
\Delta R^{i}=\operatorname{diag}\{0, \ldots, 0, \pm \gamma, 0, \ldots, 0, \mp \gamma, 0, \ldots ., 0\}
$$

e $\gamma$ é um número positivo de valor elevado.

A matriz de modificações $\Delta R^{i}$ da Eq. (17), de dimensão $n m r \times n m r$, pode ser obtida pelo produto de matrizes:

$$
\Delta R^{i}=E_{i} G_{i} E_{i}^{T}
$$

onde:

$$
E_{i}=\left(e_{i_{1}} \ldots e_{i_{k}}\right)
$$

e:

$e_{i_{j}}$ : j-ésima coluna da matriz identidade;

$G_{i}$ : matriz diagonal $2 k \times 2 k$ com valores $\pm \gamma \mathrm{e}$

$k$ : é o número de disjuntores suspeitos cujo status é diferente em $\mathcal{H}_{i}$ com relação a $\mathcal{H}_{0}$.

\subsection{Testes de Hipóteses via Teorema de Bayes}

O propósito dos testes de hipóteses é estabelecer se as informações obtidas, sejam elas provenientes de medidas ou restrições, dão respaldo à hipótese básica ou a alguma das hipóteses alternativas.

A estimação Bayesiana será utilizada para calcular as probabilidades condicionais de cada hipótese. Seja $P\left(\mathcal{H}_{i}\right)$ a probabilidade a priori da hipótese $i$ e $P\left(\mathcal{H}_{i} \mid z\right)$ a probabilidade condicional a posteriori. $P\left(\mathcal{H}_{i}\right)$ deve ser fornecida como dado de entrada. Na falta de melhor sugestão, pode-se admitir que todas as hipóteses são equiprováveis. A forma relevante do Teorema de Bayes para a presente aplicação estabelece que (Papoulis, 1991):

$$
P\left(\mathcal{H}_{i} \mid z\right)=\frac{f\left(z \mid \mathcal{H}_{i}\right) P\left(\mathcal{H}_{i}\right)}{\sum_{j=1}^{2^{n} d s} f\left(z \mid \mathcal{H}_{j}\right) P\left(\mathcal{H}_{j}\right)}
$$

onde $f\left(z \mid \mathcal{H}_{i}\right)$ é a função densidade probabilidade condicional de $z$ dado que $\mathcal{H}_{i}$ é verdadeiro.

Considerando-se que as variáveis de estados $x$ e os erros de medição $\varepsilon$ sejam variáveis aleatórias normalmente distribuídas, suas funções densidade podem ser expressas por (Papoulis, 1991):

$$
f_{x}(x)=2 \pi^{-\frac{n}{2}} \sqrt{\left|P^{-1}\right|} e^{\left\{-\frac{1}{2} x^{T} P^{-1} x\right\}}
$$

e

$$
f_{\epsilon}\left(\epsilon \mid \mathcal{H}_{i}\right)=2 \pi^{-\frac{m}{2}}\left|R_{i}\right|^{-\frac{1}{2}} e^{\left\{-\frac{1}{2} \epsilon^{T} R_{i}^{-1} \epsilon\right\}}
$$

$\mathrm{O}$ vetor de medidas/restrições $z$ pode ser expresso em função do vetor de estados por:

$$
z=H x+\epsilon
$$

onde $\varepsilon$ é o vetor de erros, de dimensões $n m r \times 1$ :

$$
\epsilon=\left(\begin{array}{ccc}
\varepsilon_{m} & 0 & 0
\end{array}\right)^{T}
$$

Assim, a função densidade probabilidade condicional para cada hipótese $i, f\left(z \mid \mathcal{H}_{i}\right)$, também será gaussiana (Papoulis, 1991) e pode ser expressa por:

$$
f\left(z \mid \mathcal{H}_{i}\right)=2 \pi^{-\frac{m}{2}}\left|\Omega_{i}\right|^{-\frac{1}{2}} e^{\left\{-\frac{1}{2}(z-H \bar{x})^{T} \Omega_{i}^{-1}(z-H \bar{x})\right\}}
$$

onde $\Omega_{i}$ é a matriz de covariância do vetor $z$ considerando que a hipótese $\mathcal{H}_{i}$ seja verdadeira, de dimensões $n m r \times n m r$, dada por:

$$
\Omega_{i}=R_{i}+H P H^{T}
$$

O cálculo do determinante $\left|\Omega_{i}\right|$ pode ser realizado eficientemente através da aplicação de algumas propriedades de determinantes e do teorema de Sherman-MorrisonWoodbury (Golub et alli, 1996). 


\section{OBSERVAÇÕES SOBRE A APLICAÇÃO DO MÉTODO PROPOSTO}

O método proposto neste trabalho utiliza a modelagem no nível de substação para a rede elétrica. Com isto, os disjuntores das substações suspeitas são explicitamente representados no modelo da rede, o que facilita a identificação de erros de topologia. No entanto, conforme discutido em (Clements e Simões Costa, 1997) e (Lourenço, 2001), este nível de detalhamento torna frequente a ocorrência de ilhamentos e a incidência de informações críticas e conjuntos críticos envolvendo restrições operacionais. Ambos os problemas podem inviabilizar o processamento de erros de topologia. Com relação ao primeiro caso, as diferentes ilhas surgidas com o detalhamento da rede (seções de barra isoladas, por exemplo) não podem ser tratadas independentemente, já que estas ilhas podem ser conseqüência de erros no status de um ou mais disjuntores. As informações a priori relativas aos ângulos nas barras, modeladas de acordo com a Seção 2.4, permitem que a estimação de estados possa ser realizada para sistemas compostos por diferentes ilhas, sem que haja a necessidade de definir uma barra de referência para cada uma delas, uma vez que estas informações atuam como medidas virtuais para os ângulos das barras do sistema.

A discussão acima nos permite concluir que a utilização das informações a priori sobre os estados equivale a um aumento da redundância fornecida pelas medidas e restrições disponíveis no sistema. Conseqüentemente, as dificuldades causadas por informações (medidas e restrições) críticas e por conjuntos críticos são reduzidas quando se utiliza informações a priori. De fato, verificou-se a eficácia do método proposto na identificação de erros de topologia mesmo na ocorrência de conjuntos críticos entre os disjuntores envolvidos. Entretanto, níveis muito baixos de redundância ainda podem vir a prejudicar a inclusão de todos os disjuntores erroneamente modelados no conjunto dos suspeito (ver subseção 3.2), comprometendo a identificação dos erros através dos testes de hipóteses. Um teste geométrico foi desenvolvido recentemente (Lourenço et alli, 2002), o qual garante a inclusão dos disjuntores erroneamente modelados no conjunto suspeito, mesmo em casos de baixa redundância, aumentando a viabilidade prática do método proposto neste artigo.

Outro problema que pode comprometer o desempenho do método proposto é a ocorrência de erros grosseiros em medidas, os quais podem ser inadvertidamente identificados como erros de topologia. Pesquisas adicionais estão sendo desenvolvidas neste sentido, com o objetivo de estender a metodologia proposta para possibilitar a análise simultânea de erros grosseiros em medidas e erros de topologia.

\section{RESULTADOS}

Os resultados desta seção foram obtidos utilizando-se o mesmo sistema-teste de 30 barras do IEEE, tendo sido simulados os mesmos casos de erros topológicos apresentados em (Clements e Simões Costa, 1997). Considerase que as substações a serem detalhadas, definidas pelo pré-processamento, correspondem às barras 12 e 15 do sistema original, aqui renumeradas como barras 2 e 5 , respectivamente. A porção relevante do sistema-teste é mostrada na Figura 1 e compreende as substações suspeitas modeladas no nível de seção de barra.

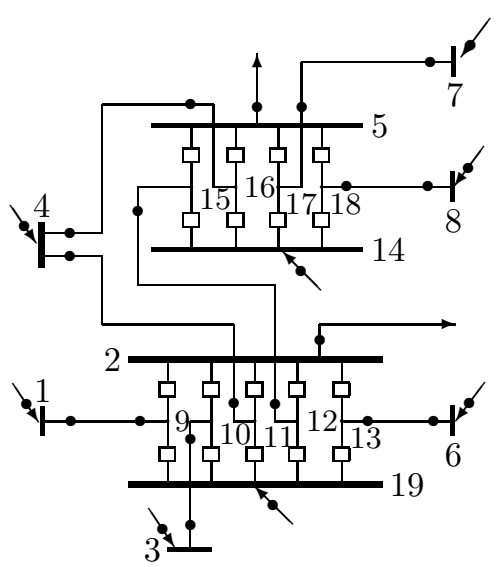

Figura 1: Sistema Teste - 19 nós, 26 ramos

As Tabelas 1 e 2 mostram as condições de operação e os planos de medição utilizados para o sistema-teste. O limiar $\lambda_{t}$ foi estabelecido em 3,0 para para todos os casos simulados.

Tabela 1: Condições de Operação para o Sistema Teste

\begin{tabular}{|l||l|}
\hline \hline \multicolumn{2}{|c|}{ Disjuntores Fechados } \\
\hline \hline \multicolumn{1}{|c|}{ CO-1 } & \multicolumn{1}{c|}{ CO-2 } \\
\hline \hline $2-9,2-10,2-11,2-13$, & $2-9,2-10,2-11,2-12,2-13$, \\
$5-16,5-17,5-18$ & $5-15,5-16,14-17,14-18$ \\
\hline \hline
\end{tabular}

Tabela 2: Planos de Medição para o Sistema Teste

\begin{tabular}{|l||l|}
\hline \hline Plano de Med. 1 & \multicolumn{1}{|c|}{ Plano de Med. 2 } \\
\hline \hline $2-9,2-10,2-13$, & $2-9,2-10,2-13,5-15,5-16$, \\
$5-18,19-12$, & $5-17,5-18,19-12,14-16$, \\
$14-16,14-17$ & $14-17,14-18,14-15$, \\
\hline \hline
\end{tabular}




\subsection{Erros Simples}

Esta subseção apresenta os resultados dos testes onde o status de um único disjuntor é erroneamente reportado para o estimador de estados. Utilizou-se a condição de operação CO-1 e o plano de medição 1, mostrados nas Tabelas 1 e 2, respectivamente.

Nos três casos simulados, o teste dos multiplicadores de Lagrange normalizados indica a presença do erro de topologia, e portanto o processamento de erros topológicos tem continuidade para identificar os disjuntores cujo status está incorreto. Os disjuntores selecionados como suspeitos e os resultados obtidos com os testes de hipóteses estão mostrados na Tabela 3. Verifica-se que, para os três casos simulados, o valor de $\lambda_{o}^{N}$ referente ao disjuntor erroneamente modelado é o único com magnitude superior ao limiar $\lambda_{t}$. O conjunto de disjuntores suspeitos se restringe, portanto, ao disjuntor erroneamente modelado, simplificando a aplicação dos testes de hipóteses. A hipótese básica é definida pela configuração atual, ou seja, pela posição aberta do disjuntor suspeito $\left(\mathcal{H}_{0}:\{0\}\right)$. $\mathrm{O}$ valor da probabilidade condicional para a única hipótese alternativa $\mathcal{H}_{1}$ (referente ao status fechado, $\left.\mathcal{H}_{1}:\{1\}\right)$, mostrado na Tabela 3 , é igual a 1,0 , indicando que esta representa a correta configuração do disjuntor suspeito em cada caso simulado.

Tabela 3: Resultados para Erros Simples

\begin{tabular}{|c|c|c|c|}
\hline \hline $\begin{array}{c}\text { Disj. Erron. } \\
\text { Modelados }\end{array}$ & $\begin{array}{c}\text { Disj. Susp. } \\
\left(\lambda_{o}^{N}>\lambda_{t}\right)\end{array}$ & $\mathcal{H}_{i}$ & $P\left(\mathcal{H}_{i} \mid z\right)$ \\
\hline \hline Caso 1: 5-16 & $5-16$ & $\mathcal{H}_{1}:\{1\}$ & 1.00 \\
\hline Caso 2: 2-13 & $2-13$ & $\mathcal{H}_{1}:\{1\}$ & 1.00 \\
\hline Caso 3: 2-10 & $2-10$ & $\mathcal{H}_{1}:\{1\}$ & 1.00 \\
\hline \hline
\end{tabular}

\subsection{Erro de Inclusão}

O erro de inclusão simulado refere-se ao ramo 12-15, o qual não está em operação mas é inadvertidamente incluído no modelo da rede. Para simular este erro, os status dos disjuntores 2-12 e 5-15 são supostos fechados. O plano de medição 1 e a condição de operação 1 das Tabelas 2 e 1 são empregados para o estimador e para o processamento de erros topológicos.

Os disjuntores selecionados como suspeitos pelo método estão mostrados na Tabela 4 . Na terceira e quarta colunas da mesma Tabela são mostradas as combinação que representam as configurações correta e simulada do conjunto de disjuntores suspeitos, respectivamente.

Os resultados obtidos com a aplicação dos testes de hipóteses para as hipóteses alternativas cujas probabilida-
Tabela 4: Disjuntores Suspeitos - Erro de Inclusão

\begin{tabular}{|c||c|c|c|}
\hline \hline $\begin{array}{c}\text { Disj. Erron. } \\
\text { Modelados }\end{array}$ & $\begin{array}{c}\text { Disj. Susp. } \\
\left(\lambda_{o}^{N}>\lambda_{t}\right)\end{array}$ & $\begin{array}{c}\text { Status } \\
\text { Correto }\end{array}$ & $\begin{array}{c}\text { Status } \\
\text { Simulado }\left(\mathcal{H}_{0}\right)\end{array}$ \\
\hline \hline & $2-11$ & 1 & 1 \\
$2-12$ & $2-12$ & 0 & 1 \\
$5-15$ & $5-15$ & 0 & 1 \\
& $5-16$ & 1 & 1 \\
\hline \hline
\end{tabular}

des condicionais resultaram em valores não nulos, são apresentados na Tabela 5. Verifica-se que as combinações dos status referentes a estas hipóteses correspondem exatamente às três possíveis configurações onde o ramo 12-15 estaria fora de operação, indicando que esta é a configuração correta para este ramo. Os disjuntores 2-11 e 5-16, apesar de considerados suspeitos, não estão erroneamente modelados. Este fato é confirmado pelas três combinações mostradas na Tabela 5, que indicam a posição original (fechada) como sendo a correta configuração destes disjuntores.

Tabela 5: Resultados dos Testes de Hipóteses - Erro de Inclusão

\begin{tabular}{|cc|c|c|}
\hline \hline \multicolumn{2}{|c|}{ Combinações $\left(\mathcal{H}_{i}\right)$} & $P\left(\mathcal{H}_{i}\right)$ & $P\left(\mathcal{H}_{i} \mid z\right)$ \\
\hline \hline $\mathcal{H}_{8}:$ & 1001 & 0.1536 & 0.1587 \\
$\mathcal{H}_{13}:$ & 1101 & 0.4096 & 0.4233 \\
$\mathcal{H}_{14}:$ & 1011 & 0.4096 & 0.4180 \\
\hline \hline
\end{tabular}

\subsection{Erro do Tipo Bus Split}

A condição de operação para este caso é CO-2, apresentada na Tabela 1, e o plano de medição utilizado é o plano 2 da Tabela 2. É fácil verificar que a configuração resultante de CO-2 implica em um seccionamento de barra na substação representada na parte superior da Figura 1. Entretanto, este seccionamento é ignorado no modelo da rede, o qual supõe erroneamente que os disjuntores 5-17 e 5-18 estão fechados enquanto os disjuntores 14-17 e 14-18 estão abertos.

Pode-se verificar que, tanto para a configuração real quanto para a simulada, dois disjuntores estarão radialmente conectados aos ramos 7-17 e 7-18. Conforme discutido em (Clements e Simões Costa, 1997), configurações radiais envolvendo disjuntores tornam seus status críticos e portanto não detectáveis. O mesmo procedimento adotado em (Clements e Simões Costa, 1997) foi aplicado, segundo o qual o sistema-teste é expandido através da representação das barras denotadas por $7^{\prime} \mathrm{e}$ $8^{\prime}$, de forma a incluir o laço mostrado na Figura 2. 


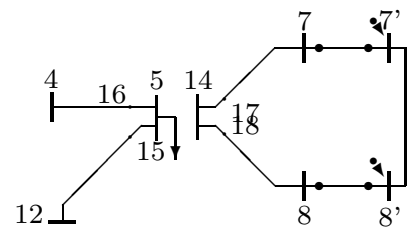

Figura 2: Laço para Sistema Teste Estendido

A Tabela 6 mostra os disjuntores selecionados como suspeitos pelo estimador. Neste caso, o conjunto formado por esses disjuntores corresponde examente ao conjunto de disjuntores erroneamente modelados. Os valores não nulos das probabilidades condicionais fornecidos pelos testes de hipóteses são mostrados na Tabela 7. O maior valor obtido para a probabilidade condicional corresponde à hipótese alternativa $\mathcal{H}_{6}(1100)$, que representa a configuração correta do conjunto de disjuntores suspeitos. Comparando a hipótese básica com a hipótese alternativa $\mathcal{H}_{6}$, confirma-se a existência do erro de modelagem envolvendo os 4 disjuntores suspeitos e o erro de bus-split é corretamente identificado.

Tabela 6: Disjuntores Suspeitos - Erro Bus Split

\begin{tabular}{|c||c|c|c|}
\hline \hline $\begin{array}{c}\text { Disj. Erron. } \\
\text { Modelados }\end{array}$ & $\begin{array}{c}\text { Disj. Susp. } \\
\left(\lambda_{o}^{N}>\lambda_{t}\right)\end{array}$ & $\begin{array}{c}\text { Status } \\
\text { Correto }\end{array}$ & $\begin{array}{c}\text { Status } \\
\text { Simulado }\left(\mathcal{H}_{0}\right)\end{array}$ \\
\hline \hline $14-17$ & $14-17$ & 1 & 0 \\
$14-18$ & $14-18$ & 1 & 0 \\
$5-17$ & $5-17$ & 0 & 1 \\
$5-18$ & $5-18$ & 0 & 1 \\
\hline \hline
\end{tabular}

\subsection{Erro de Exclusão}

A condição de operação CO-2 da Tabela 1 e o plano de medição 2 da Tabela 2 são novamente empregados neste caso. No erro de exclusão simulado os disjuntores 2-12 e 5-15 são considerados abertos pelo estimador, o que exclui erroneamente o ramo 12-15 do modelo da rede.

O conjunto de disjuntores suspeitos obtido a partir dos multiplicadores de Lagrange normalizados associados às restrições operacionais é formado pelos disjuntores $\{5$ -

Tabela 7: Resultados dos Testes de Hipóteses - Erro bus-split

\begin{tabular}{|cc|c|c|}
\hline \hline \multicolumn{2}{|c|}{$\mathcal{H}_{i}$} & $P\left(\mathcal{H}_{i}\right)$ & $P\left(\mathcal{H}_{i} \mid z\right)$ \\
\hline \hline $\mathcal{H}_{6}:$ & 1100 & 0.0016 & 0.8978 \\
$\mathcal{H}_{11}:$ & 1110 & 0.0256 & 0.0352 \\
$\mathcal{H}_{12}:$ & 1101 & 0.0256 & 0.0653 \\
$\mathcal{H}_{15}:$ & 1111 & 0.1536 & 0.0018 \\
\hline \hline
\end{tabular}

18, 5-17, 11-19, 14-16, 9-19, 10-19, 13-19\}. Observa-se portanto que os disjuntores erroneamente modelados, 212 e 5-15, não estão incluídos neste conjunto. Consistentemente, os resultados obtidos com a aplicação dos testes de hipóteses fornecem probabilidades condicionais nulas para todas as hipóteses alternativas. Isto é, os testes de hipóteses indicam corretamente que, para o conjunto de disjuntores selecionados como suspeitos, as informações fornecidas para o estimador sustentam a hipótese básica, segundo a qual estes disjuntores estão corretamente modelados.

Verifica-se portanto que a não-ocorrência dos disjuntores erroneamente modelados entre aqueles originalmente classificados como suspeitos impede a identificação dos erros de topologia. Não obstante, deve ser ressaltado que os testes de hipóteses baseados no teorema de Bayes não tentam atribuir os erros de modelagem a outras combinações de disjuntores. Persistem assim os sintomas da presença do erro, sem uma identificação conclusiva de suas causas.

Conclui-se que a falha na identificação do erro de exclusão deve-se ao procedimento de seleção dos disjuntores suspeitos. Para comprovar isto, suponha que este procedimento é modificado e considera agora os multiplicadores de Lagrange normalizados associados às medidas $\left(\lambda_{m}^{N}\right)$ próximas aos disjuntores, ao invés daqueles associados às restrições operacionais. $\mathrm{O}$ critério utilizado para inclusão no rol de disjuntores suspeitos é $\lambda_{m}^{N}>3,0$. Com este critério verifica-se que os disjuntores erroneamente modelados (2-12 e 5-15) são os únicos selecionados como suspeitos. A Tabela 8 resume os resultados obtidos com o procedimento modificado para seleção de disjuntores suspeitos.

Tabela 8: Disjuntores Suspeitos por $\lambda_{m}^{N}$ - Erro de Exclusão

\begin{tabular}{|c|c|c||c|}
\hline \hline $\begin{array}{c}\text { Disj. Erron. } \\
\text { Modelados }\end{array}$ & $\begin{array}{c}\text { Status } \\
\text { Correto }\end{array}$ & $\begin{array}{c}\text { Status } \\
\text { Simulado }\end{array}$ & $\begin{array}{c}\text { DS } \\
\left(\lambda_{m}^{N}>\lambda_{t}\right)\end{array}$ \\
\hline \hline $2-12$ & 1 & 0 & $2-12$ \\
$5-15$ & 1 & 0 & $5-15$ \\
\hline \hline
\end{tabular}

A aplicação dos testes de hipóteses considerando a lista de disjuntores suspeitos da Tabela 8 resulta em uma probabilidade condicional igual a 1,0 para a hipótese alternativa $\mathcal{H}_{3}:\{11\}$, indicando que esta é a configuração correta dos disjuntores suspeitos. As probabilidades condicionais obtidas para as outras duas hipóteses alternativas $\left(\left\{\begin{array}{ll}0 & 1\end{array}\right\}\right.$ e $\left.\left\{\begin{array}{ll}1 & 0\end{array}\right\}\right)$ foram ambas nulas.

Utilizar os multiplicadores de Lagrange normalizados associados às medidas, conforme apresentado acima, não 
é uma alternativa válida para a seleção de disjuntores suspeitos, e não se constitui em uma proposta deste artigo. Há pelo menos duas boas razões para isto. A primeira diz respeito à necessidade de haver medidas no plano de medição associadas diretamente ao ramo chaveável envolvido no erro topológico. A segunda razão relaciona-se à possibilidade real de ocorrência de erros grosseiros em medidas, que poderiam ser indevidamente tratados como erros de topologia.

O que os resultados acima de fato demonstram é a necessidade de aprimoramento do método de seleção de disjuntores suspeitos. Neste sentido, estão presentemente sendo avaliadas duas vertentes: (i) o uso das análises generalizadas de observabilidade e criticidade de medidas e restrições como ferramenta acessória àquela seleção, e (ii) a extensão do método baseado em diferenças angulares apresentado em (Clements e Davis, 1983), visando sua aplicação como uma ferramenta alternativa para seleção de disjuntores suspeitos.

\section{CONCLUSÕES}

Este trabalho propõe o uso de testes de hipóteses baseados em estatística bayesiana para a identificação de erros de topologia na modelagem em tempo real de sistemas de potência. O método proposto foi testado considerando os diferentes tipos de erros de topologia: erro de configuração de um único disjuntor, erros de inclusão e exclusão de ramos da rede e erros do tipo bus split em substações. Os resultados obtidos mostram que, se a redundância do plano de medição e a topologia da rede permitem a inclusão dos disjuntores incorretamente modelados no conjunto de disjuntores suspeitos, a configuração correta destes disjuntores é identificada através das probabilidades condicionais a posteriori fornecidas pelo Teorema de Bayes.

O método proposto apresenta como principal vantagem o fato de não exigir re-estimações de estados para cada configuração alternativa dos disjuntores da rede. Ao invés disso, faz uso apenas da estimação de estados correspondente à configuração corrente, a partir da qual são determinados todos os dados necessários para a análise das hipóteses alternativas.

\section{AGRADECIMENTOS}

Os autores gostariam inicialmente de agradecer as valiosas sugestões dos revisores do artigo submetido ao XIII CBA. Adicionalmente, Elizete Maria Lourenço agradece o suporte financeiro da Universidade Federal do Paraná e da CAPES para a realização de seu curso de Douto- rado na Universidade Federal de Santa Catarina. Antonio Simões Costa também expressa seus agradecimentos ao $\mathrm{CNPq}$, pelo suporte financeiro a este trabalho de pesquisa.

\section{REFERÊNCIAS}

Alsaç, O., Vempati, N., Stott, B. e Monticelli, A. (1998). Generalized State Estimation. Transactions on Power Systems 13(3): 1069-1075.

Clements, K.A. e Davis, P.W. (1983). Multiple Bad Data Detectability and Identifiability: A Geometric Approach. Transactions on Power Systems 13(2): 461-466.

Clements, K.A. e Simões Costa, A. (1997). Topology Error Identification Using Normalized Lagrange Multipliers. Transactions on Power Systems 13(2): 347353.

Gjelsvik, A., Aam, A. e Holten, L. (1985). Aumented Matrix Method: A Rapid Method for Improving Numerical Stability in Power System State Estimation. Transactions on Power Application and Systems 104(6): 2987-2993.

Gjelsvik, A. (1993). The Significance of the Lagrange Multipliers in WLS State Estimation with Equality Constraints. Proceedings of the 11th Power Systems Computation Conference, pp. 619-625.

Singh, N., Glavitsch, H. (1991). Detection and Identification of Topological Errors in Online Power System Analysis. IEEE Transactions on Power Systems (6): 324-331.

Singh, N., Oesch, F. (1994). Pratical Experience with Rule-Based On-Line Topology Error Detection. IEEE Transactions on Power Systems (9): 841-847.

Souza, J. C. S., Leite da Silva, A. M., Alves da Silva, A.P. (1998). Online Topology Determination and Bad Data Supression in Power System Operation Using Artificial Neural Network. IEEE Transactions on Power Systems 13(3): 796-803.

Abur, A., Kim, H., Celik, M. K. (1995). Identifying the Unknown Circuit Breaker Status in Power Network. IEEE Transactions on Power Systems 10(4): 20292037.

Golub, G. H. e Van Loan, C. F. (1996). Matrix Computation. 3rd ed. The John Hopikins University Press, Baltimore. 
Monticelli, A. e Garcia, A. (1991). Modeling Zero Impedance Branches in Power System State Estimation. IEEE/PES Winter Meeting 8(1): 364-370.

Monticelli, A. (1993). Modeling Circuit Breakers in Weighted Least Squares State Estimation. Transactions on Power Systems 8(3): 1143-1149.

Papoulis, A. (1991). Probability, Random Variables and Stochastic Processes. 3rd ed. McGraw Hill.

Lourenço, E. M., Clements K. A. e Simões Costa, A. J. A. (2002). Geometrically-Based Hypothesis Testing for Topology Error Identification. 14th Power Systems Computation Conference Sevilha, Espanha.

Lourenço, E. M. (2001). Análise de Observabilidade e Identificação de Erros de Topologia na Estimação de Estados Generalizada. Tese de Doutorado. Universidade Federal de Santa Catarina. 